\title{
Surface Segregation of CoPt Polycrystalline Alloy
}

\author{
P.J. Godowski ${ }^{a, *}$, T. OHGI ${ }^{b}$ AND D. FuJita ${ }^{b}$ \\ ${ }^{a}$ Institute of Experimental Physics, University of Wrocław \\ pl. Maxa Borna 9, 50-204 Wrocław, Poland \\ ${ }^{b}$ Nanomaterials Laboratory, National Institute for Materials Science \\ 1-2-1 Sengen, Tsukuba, Ibaraki 305-0047, Japan
}

(Received March 28, 2003; in final form June 9, 2003)

\begin{abstract}
Composition-depth profiling using different emission angles of X-ray photoelectron spectroscopy showed surface enhancement of platinum concentration of the $\mathrm{CoPt}(30 \mathrm{at} \%)$ alloy extensively annealed to $1000 \mathrm{~K}$ in ultrahigh vacuum. The results are compared with theoretical predictions basing on models with no crystal face dependence.
\end{abstract}

PACS numbers: 68.35.Dv, 68.47.De, 79.60.-i

\section{Introduction}

Segregation and chemical ordering on the alloy surfaces were extensively investigated in the past and comprehensive reviews have appeared [1-3]. The knowledge of the surface composition and structure of the alloy catalyst allows the prediction of the given chemical reaction behavior (enhancement or suppression due to the presence of a specific intercalation) and is of special importance. Cobalt based alloys play a significant role in industrial catalysis and this stimulates investigations of these materials. The magnetic properties of $\mathrm{CoPt}$ alloys, especially thin films, which are important in the electronics were investigated recently as well $[4,5]$. The phase diagram of the $\mathrm{Co}-\mathrm{Pt}$ system shows that the alloy crystallizes to form a continuous series of solid solutions. In the region of 50 at $\%$ and 75 at $\%$ of $\mathrm{Pt}$ the disorder-order transformations exist giving ordered fcc superlattice treated as intermetallic system [6].

*corresponding author; e-mail: pjg@ifd.uni.wroc.pl 
As concerns surface analysis of the CoPt alloy the following volume compositions have been experimentally studied: $\operatorname{CoPt}(20 \mathrm{at} \%)$ of the (110) and (111) single crystal surfaces [7], $\operatorname{CoPt}(25$ and 35 at\%) of the (110) face [8], $\operatorname{CoPt}(75$ at\%) of the (100) [9], (110) [10] and (111) face [11]. It was determined that segregation is face dependent, with Pt segregated at the (111) and (100) faces and Co at the (110) face.

It was interesting to investigate the segregation behavior of the polycrystalline alloy of the volume composition complementary to the above mentioned cases, i.e. of $\operatorname{CoPt}(30 \mathrm{at} \%)$. Polycrystals are more close to the thermodynamic models of the process as well as to some real catalysts (e.g. grids). Determination of the surface composition and structure is important in order to predict a result of a catalytic reaction. It is known that under some circumstances X-ray induced photoelectron spectroscopy (XPS) is able to provide information about the chemical states of the elements and their relative contents. In this study angle-resolved photoelectron spectroscopy (AR-XPS) was applied in evaluation of concentration gradient normal to the surface.

\section{Experimental}

Experiments were performed using an ultrahigh vacuum (UHV) chamber equipped with the hemispherical sector spectrometer of the ARUPS 10 type (VG Scientific). The base pressure of the chamber during measurements was lower than $1 \times 10^{-9}$ mbar. X-ray source with $\mathrm{Mg}$ anode could operate at $15 \mathrm{kV}$ and $30 \mathrm{~mA}$ giving $\mathrm{Mg} K_{\alpha}$ radiation (the mean energy of $1253.53 \mathrm{eV}$ )[12] of high intensity. The analyzer input lens were set to mode 1 giving lens half angle of $1.2^{\circ}$ and/or occasionally to mode $2\left(0.8^{\circ}\right)$. The spatial uniformity of the X-ray flux (high flux and large irradiated area) and the used input lens fulfill good conditions for obtaining proper AR-XPS profiles. Binding energies, referenced to the sample Fermi energy, were calibrated using $\mathrm{Au} 4 f_{7 / 2}$ photoelectrons at $84.0 \mathrm{eV}$ and $\mathrm{Cu}$ $2 p_{3 / 2}$ photoelectrons at $932.7 \mathrm{eV}$ [13]. The spectrometer was interfaced to an IBM PC microcomputer for data acquisition. Peak analysis was accomplished based on the Shirley background subtraction and mixed Gaussian-Lorentzian peaks using curve fitting program of Kwok [14]. Several analysis were performed with fitting to the Doniach-Sunjic line shape using program of FitXPS [15].

The CoPt alloy (melting point of Co is $1768 \mathrm{~K}$ and $\mathrm{Pt} 2041 \mathrm{~K}$ ) was prepared from high purity powders (Johnson-Matthey Chemicals, Ltd.) by sintering and homogenization in an inert atmosphere $\left(\mathrm{N}_{2}\right)$ at around $1750 \mathrm{~K}$ over $10 \mathrm{~h}$. X-ray diffraction analysis of the obtained ingot confirmed the homogeneity of the alloy. A specimen slice of $c a .10 \mathrm{~mm}$ in diameter and $0.8 \mathrm{~mm}$ thick was cut from the ingot and mechanically polished with diamond paste to obtain mirror-like surfaces. The sample was mounted on the VG holder which included sample heating option. The alloy surface was cleaned in UHV by repeated Ar ion bombardment $(1 \mathrm{kV}$, 
$5 \times 10^{-6}$ mbar, $\left.5 \mu \mathrm{A}\right)$ and annealed until there was no impurity peaks $(\mathrm{C}, \mathrm{P}, \mathrm{S})$ detected by XPS.

\section{Results and discussion}

To obtain the segregation equilibrium in the subsurface region of the alloy, the sample was annealed in UHV at $1000 \mathrm{~K}$ for above 50 hours. Then XPS analysis was performed at room temperature. Wide-range photoemission spectrum as a function of kinetic energy of photoelectrons of the $\operatorname{CoPt}(30 \mathrm{at} \%)$ alloy obtained after annealing is shown in Fig. 1.

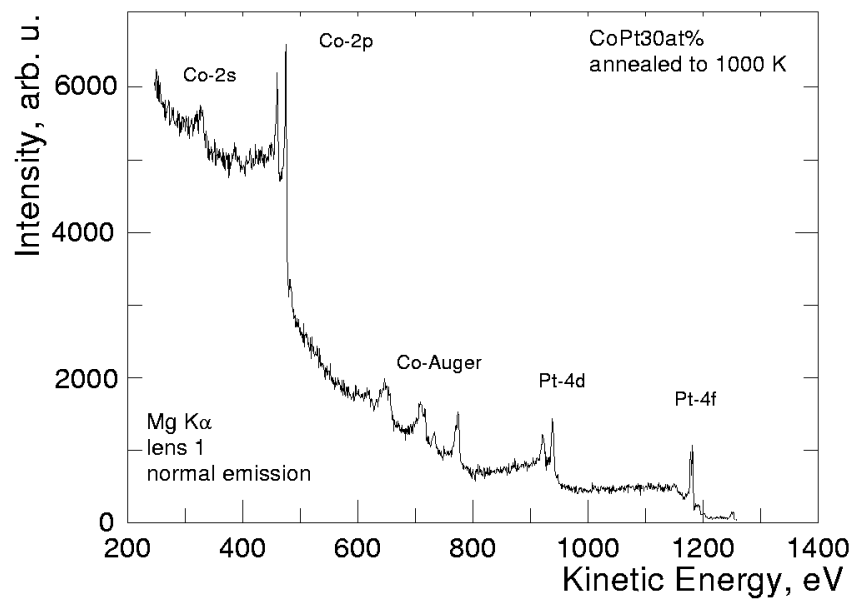

Fig. 1. Wide-range XPS spectrum of the CoPt(30at\%) alloy annealed in UHV at $1000 \mathrm{~K}$.

The most pronounced features observed in the spectrum correspond to the Co- $2 s$, the Co- $2 p$, the Co-Auger, the $\mathrm{Pt}-4 d$, and the $\mathrm{Pt}-4 f$ level. Taking into account the average monolayer thickness, $a$, of the alloy in the subsurface region as $a_{\mathrm{CoPt}}=0.7 a_{\mathrm{Co}}+0.3 a_{\mathrm{Pt}}=0.7 \times 0.2224 \mathrm{~nm}+0.3 \times 0.2472 \mathrm{~nm}=0.2298 \mathrm{~nm}$ into the Seah-Dench formula (for elements) on the attenuation length (AL) of photoelectrons in the alloy we obtain $0.816,0.985,1.26,1.38$, and $1.55 \mathrm{~nm}$ indicating the sampling depth corresponding to the above peaks, respectively [16]. High-resolution spectra of the Co- $2 p$, the Co-Auger, the $\mathrm{Pt}-4 d$, and the $\mathrm{Pt}-4 f$ were taken for nine angles, in $10^{\circ}$ interval between normal emission $\left(\theta=90^{\circ}\right)$ and $\theta=20^{\circ}$ and last spectra at $\theta=15^{\circ}$, where $\theta$ is the take-off angle measured with respect to the surface plane.

The fitting results of the analyzed photoelectron peaks are shown in Table I; the binding energy (BE) scale refers to the sample Fermi energy. The first row (top and bottom table) includes the database data of the pure element of cobalt and platinum [17] and the binding energies could be compared. The uncertainties, 
TABLE I

Parameters of the Co- $2 p$, the Co-Auger, the $\mathrm{Pd}-4 d$ and the $\mathrm{Pt}-4 f$ peaks of the pure metal and the annealed at $1000 \mathrm{~K} \mathrm{CoPt}(30 \mathrm{at} \%)$ alloy for different take-off angle. All level energies, BE, are referenced to the sample Fermi energy and expressed in $\mathrm{eV}$. Intensity, $I$, the area under the peak, is expressed in arbitrary units. Electron escape depth, ED, calculated as $\mathrm{ED}=\mathrm{AL} \sin \theta$, is expressed in $\mathrm{nm}$ and average alloy monolayers, $\mathrm{ML}$ (nm/ML).

\begin{tabular}{|c|c|c|c|c|c|c|}
\hline & \multicolumn{3}{|c|}{$\mathrm{Co}-2 p$} & \multicolumn{3}{|c|}{ Co-Auger } \\
\hline & $\mathrm{BE}$ & $I$ & ED & $\mathrm{BE}$ & $I$ & ED \\
\hline pure & & & & & & \\
\hline element & $778.18 \pm 0.25^{a}$ & & & & & \\
\hline $90^{\circ}$ & 778.23 & 2879 & $0.985 / 4.3$ & 480.1 & 1724 & $1.26 / 5.5$ \\
\hline $80^{\circ}$ & 778.24 & 2901 & $0.970 / 4.2$ & 480.0 & 1824 & $1.24 / 5.4$ \\
\hline $70^{\circ}$ & 778.30 & 3802 & $0.926 / 4.0$ & 480.1 & 1585 & $1.18 / 5.2$ \\
\hline $60^{\circ}$ & 778.32 & 3589 & $0.853 / 3.7$ & 480.5 & 1399 & $1.09 / 4.8$ \\
\hline $50^{\circ}$ & 778.36 & 3412 & $0.755 / 3.3$ & 480.2 & 1079 & $0.962 / 4.2$ \\
\hline $40^{\circ}$ & 778.42 & 3011 & $0.633 / 2.8$ & 480.2 & 1129 & $0.807 / 3.5$ \\
\hline $30^{\circ}$ & 778.51 & 1731 & $0.493 / 2.2$ & 480.6 & 1094 & $0.628 / 2.8$ \\
\hline $20^{\circ}$ & 778.79 & 703 & $0.337 / 1.5$ & 480.9 & 928 & $0.430 / 1.9$ \\
\hline \multirow[t]{3}{*}{$15^{\circ}$} & 778.83 & 412 & $0.255 / 1.1$ & 480.9 & 638 & $0.325 / 1.4$ \\
\hline & \multicolumn{3}{|c|}{$\mathrm{Pt}-4 d$} & \multicolumn{3}{|c|}{$\mathrm{Pt}-4 f$} \\
\hline & $\mathrm{BE}$ & $I$ & ED & $\mathrm{BE}$ & $I$ & ED \\
\hline pure & & & & & & \\
\hline element & $314.6^{a}$ & & & $71.11 \pm 0.14^{a}$ & & \\
\hline $90^{\circ}$ & 314.36 & 2494 & $1.38 / 6.0$ & 71.17 & 850 & $1.55 / 6.8$ \\
\hline $80^{\circ}$ & 314.33 & 2515 & $1.36 / 5.9$ & 71.18 & 884 & $1.53 / 6.7$ \\
\hline $70^{\circ}$ & 314.44 & 2707 & $1.30 / 5.6$ & 71.17 & 965 & $1.46 / 6.4$ \\
\hline $60^{\circ}$ & 314.43 & 2791 & $1.20 / 5.2$ & 71.22 & 1027 & $1.35 / 5.9$ \\
\hline $50^{\circ}$ & 314.56 & 2784 & $1.06 / 4.6$ & 71.22 & 995 & $1.19 / 5.2$ \\
\hline $40^{\circ}$ & 314.58 & 2762 & $0.890 / 3.9$ & 71.24 & 959 & $0.998 / 4.4$ \\
\hline $30^{\circ}$ & 314.61 & 2365 & $0.692 / 3.0$ & 71.20 & 756 & $0.777 / 3.4$ \\
\hline $20^{\circ}$ & 314.48 & 1612 & $0.473 / 2.1$ & 71.13 & 506 & $0.531 / 2.3$ \\
\hline $15^{\circ}$ & 314.38 & 1366 & $0.358 / 1.6$ & 71.12 & 340 & $0.402 / 1.8$ \\
\hline
\end{tabular}

${ }^{a}$ from Ref. [17]

estimated using database quantities, include systematic errors [18]. It should be mentioned that for different samples the energy zero (Fermi level) may be different with respect to some common absolute energy scale. This should be taken into consideration during comparison with the results obtained in this work. According to the electronegativity of the elements, $\mathrm{Co}(1.88)$ and $\operatorname{Pt}(2.28)$, the charge transfer from cobalt to platinum is expected. It is known that the core level BE shift, as for the noble metals, could be relatively weak, i.e. less than $1 \mathrm{eV}$ [19-21]. Taking 
the average over $90^{\circ}-50^{\circ}$ of the $\mathrm{BE}$ of the Co- $2 p$ level as the bulk data of the alloy (electron escape depth $\mathrm{ED} \geq 3 \mathrm{MLs}$ ), the value of $778.29 \pm 0.005 \mathrm{eV}$ is found (here the reproducibility of measurements [18] is indicated). The BE is shifted by $+0.11 \mathrm{eV}$ relative to the one of pure element. The shift of +0.09 is noticed for the $\mathrm{BE}$ of the Pt-4f level $\left(71.2 \pm 0.03 \mathrm{eV}\right.$, average over $\left.90^{\circ}-30^{\circ}\right)$. Above quantities of the shift lie within the uncertainty level, and it is difficult to state about charge transfer between both components of the bulk alloy. However, going to the smaller take-off angle, the relative energy shift of the Co- $2 p$ of $+0.54 \mathrm{eV}$ and the one of the $\mathrm{Pt}-4 f$ of $-0.08 \mathrm{eV}$ is observed. It is indicative that in the subsurface region (ED $<3 \mathrm{MLs}$ ) platinum enrichment takes place (greater charge transfer from Co to $\mathrm{Pt}$, i.e. greater number of $\mathrm{Pt}$ atoms). Above findings show the tendency of the formation of the CoPt phase in the topmost layers.

TABLE II

Results of the Doniach-Sunjic peak shape analysis of the Co- $2 p$, the Pt- $4 d$, and the Pt- $4 f$ level of selected take-off angles corresponding to the annealed at $1000 \mathrm{~K} \mathrm{CoPt}(30 \mathrm{at} \%)$ alloy. Fitting parameters: $\alpha$ - asymmetry parameter; BE binding energy position (referenced to the sample Fermi energy) in eV; Lfwhm - full width at half maximum of Lorentzian-shaped peak in eV. All level energies, $\mathrm{BE}$, are referenced to the sample Fermi energy and expressed in $\mathrm{eV}$.

\begin{tabular}{|c|c|c|c|c|c|}
\hline \multicolumn{6}{|c|}{ Cobalt $2 p$} \\
\hline \multirow{2}{*}{$\begin{array}{c}\text { Take-off } \\
\text { angle }\end{array}$} & \multirow[t]{2}{*}{$\alpha$} & \multicolumn{2}{|c|}{$2 p_{1 / 2}$} & \multicolumn{2}{|c|}{$2 p_{3 / 2}$} \\
\hline & & $\mathrm{BE}$ & Lfwhm & $\mathrm{BE}$ & Lfwhm \\
\hline $90^{\circ}$ & 0.52 & 793.6 & 1.85 & 778.6 & 1.15 \\
\hline $40^{\circ}$ & 0.52 & 793.7 & 1.85 & 778.7 & 1.25 \\
\hline $20^{\circ}$ & 0.50 & 794.1 & 2.14 & 779.1 & 1.29 \\
\hline \multicolumn{6}{|c|}{ Platinum $4 d$} \\
\hline \multirow{2}{*}{$\begin{array}{c}\text { Take-off } \\
\text { angle }\end{array}$} & \multirow[t]{2}{*}{$\alpha$} & \multicolumn{2}{|c|}{$4 d_{3 / 2}$} & \multicolumn{2}{|c|}{$4 d_{5 / 2}$} \\
\hline & & $\mathrm{BE}$ & Lfwhm & $\mathrm{BE}$ & Lfwhm \\
\hline $90^{\circ}$ & 0.35 & 331.6 & 4.60 & 314.7 & 3.86 \\
\hline $40^{\circ}$ & 0.39 & 331.9 & 4.42 & 315.0 & 3.66 \\
\hline $20^{\circ}$ & 0.42 & 331.8 & 5.25 & 314.9 & 4.40 \\
\hline \multicolumn{6}{|c|}{ Platinum $4 f$} \\
\hline \multirow{2}{*}{$\begin{array}{c}\text { Take-off } \\
\text { angle }\end{array}$} & \multirow[t]{2}{*}{$\alpha$} & \multicolumn{2}{|c|}{$2 p_{1 / 2}$} & \multicolumn{2}{|c|}{$2 p_{3 / 2}$} \\
\hline & & $\mathrm{BE}$ & Lfwhm & $\mathrm{BE}$ & Lfwhm \\
\hline $90^{\circ}$ & 0.16 & 74.8 & 0.99 & 71.5 & 0.90 \\
\hline $40^{\circ}$ & 0.16 & 74.9 & 0.96 & 71.6 & 0.89 \\
\hline $20^{\circ}$ & 0.17 & 74.9 & 0.96 & 71.5 & 0.86 \\
\hline
\end{tabular}


Although our instrumental resolution does not allow to measure separate bulk and surface component of Co and Pt photoemission spectra, we fit the Co and Pt peaks as the Doniach-Sunjic form [22-24]. Resulting parameters reported here (Table II) could be regarded as phenomenological values which reflect subsurface (bulk) and surface values of increasing surface contribution with decreasing take-off angle. Using the Gaussian response function from the $0.75-1.25 \mathrm{eV}$ interval, the examples of the best fits to the experimental spectra of the Co- $2 p$ and the $\mathrm{Pt}-4 f$ peak are shown in Figs. 2 and 3.

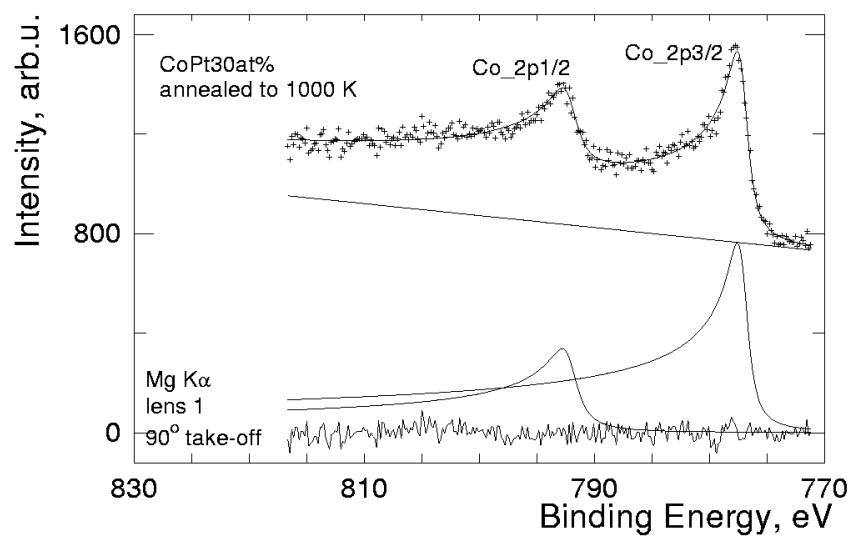

Fig. 2. Doniach-Sunjic fit to the Co- $2 p$ experimental spectrum of the annealed at $1000 \mathrm{~K} \mathrm{CoPt}(30 \mathrm{at} \%)$ alloy. A linear baseline was assumed. The points show the experimental data, the solid lines represent the best fit and the bottom line shows the residual (experimental minus fitted), which reflects the quality of the fit.

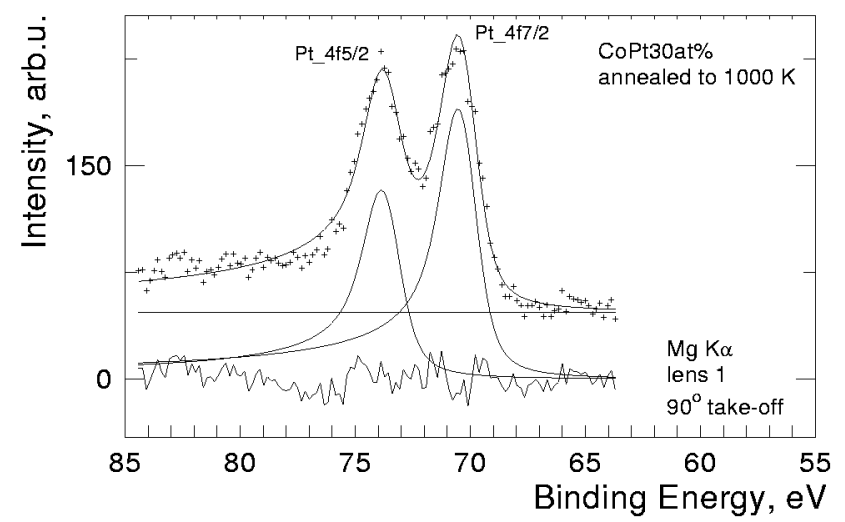

Fig. 3. Doniach-Sunjic fit to the Pt-4f experimental spectrum of the annealed at $1000 \mathrm{~K} \operatorname{CoPt}(30 \mathrm{at} \%)$ alloy. A linear baseline was assumed. The points show the experimental data, the solid lines represent the best fit and the bottom line shows the residual (experimental minus fitted), which reflects the quality of the fit. 
Because of the non-negligible noise contribution, the fit is regarded as not quite perfect one. For the $2 p, 4 d$, and $4 f$ pair peaks, the asymmetry parameter, $\alpha$, was set equal for both peaks in any given level. It is known that for the higher value of $\alpha$, the photohole is more effectively screened, corresponding to a higher local density of states (DOS) at the Fermi level. The trend in the observed value of $\alpha$ with the take-off angle is similar for both platinum levels, i.e. increase in the $\alpha$. At small take-off angles (greater surface contribution), $20^{\circ}$, the $\alpha$ decreases for Co- $2 p$ level (the most surface sensitive peak). The last behavior may be indicative for cobalt depletion at the topmost layer, giving less net charge possible to transfer to platinum there. Another fitting parameter, Lorentzian full width at half maximum (Lfwhm) is far of expectations concerning the peak half-width of metals and alloys. As was mentioned, determined values contain experimentally unresolved two: bulk and surface contributions, which concerns the widths of the photoelectron lines as well.

Inspection of Table I shows that all signals decrease with decreasing photoelectron emission angle. It can be found that the Co- $2 p$ intensity decreases decidedly more than the one of the Pt-4d. Dependences of both signals as a function of take-off angle and electron escape depths are collected in Fig. 4.

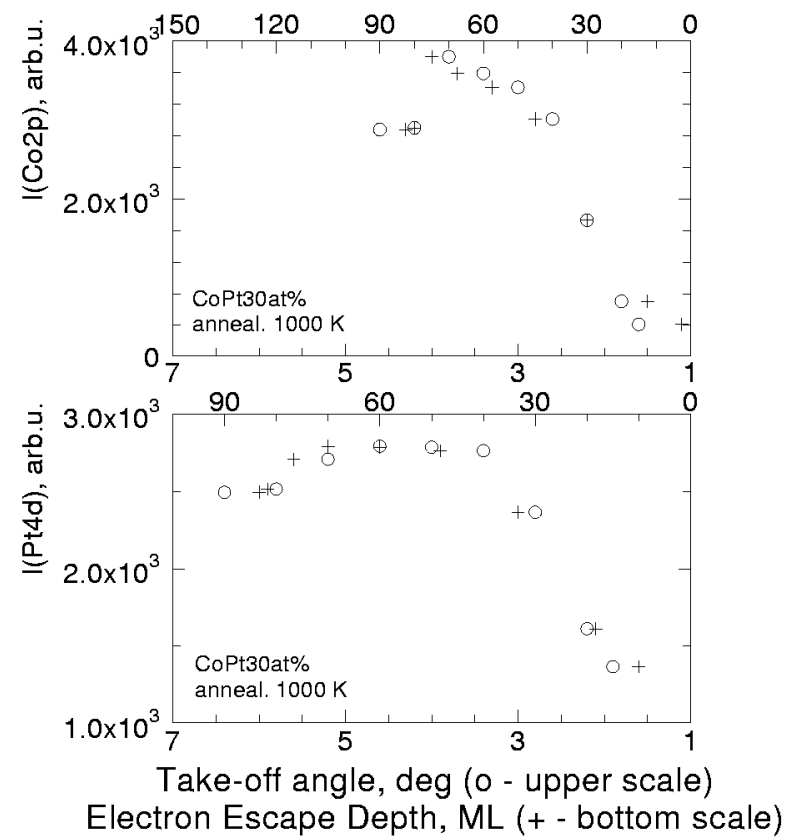

Fig. 4. Angular dependence of the Co- $2 p$ and the Pt- $4 d$ photoelectron intensities of the $\mathrm{CoPt}(30 \mathrm{at} \%)$ alloy annealed at $1000 \mathrm{~K}$. Experimental points (crosses) are drawn in the same electron escape depth range for both lines. The scales of the corresponding take-off angles (open circles data) are adjusted as to be close to the data of $(+)$. The ED is expressed in the average $30 \mathrm{at} \%$ alloy monolayers, ML. 


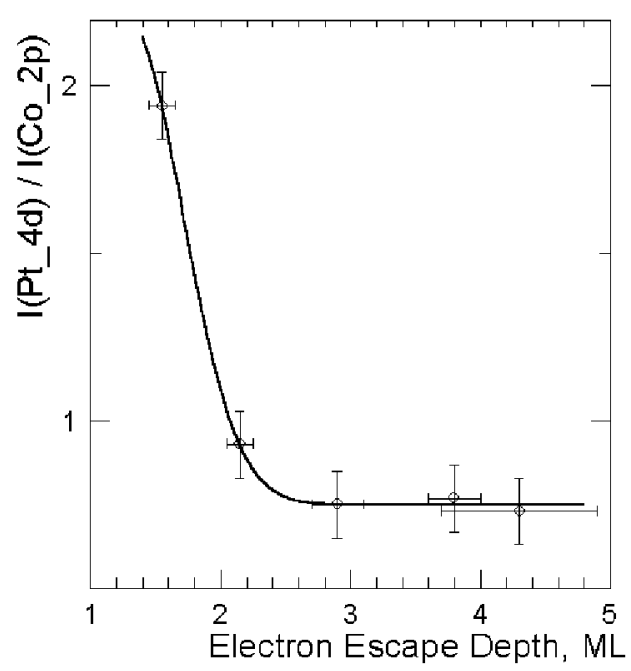

Fig. 5. Dependence of the ratio of the Pt- $4 d$ to the Co- $2 p$ photoelectron intensity with the electron escape depth as deduced from XPS angular experiment of the CoPt(30at\%) alloy annealed to $1000 \mathrm{~K}$. The increase in the ratio for ED near one monolayer indicates on increase in platinum content in the surface region.

Because of their kinetic energies, the sampling depths of both transitions are different and the proper evaluation should take this into account. To minimize instrumental errors (requirement of flat and smooth sample and decreasing count rates at glancing emission) usually the ratio techniques is utilized. The graph of the $\mathrm{Pt}-4 d$ to the Co- $2 p$ ratio as a function of the electron escape depth was constructed from the data of Table I and drawn in Fig. 5. The shape of the constructed line as the best fit to the data undoubtedly indicates the enrichment of platinum at the sample surface.

\section{Conclusions}

Comparison with theoretical predictions is restricted to the cases in which the orientation is not taken into account. Cobalt has lower melting point and the same atomic radius (Co, $135 \mathrm{pm}$; Pt, $135 \mathrm{pm}$ ), which indicates cobalt segregation. On the other hand, the Abraham-Brundle model [25], i.e. the $\gamma^{*}-\sigma^{*}$ representation of the Pt-solute in the Co-matrix (0.90-1.00) suggest platinum segregation. Predictions of Mukherjee-Moran-Lopez [26] as well as Ossi [27] shows that Co should segregate. Recent theoretical calculations by Ruban et al. [28, 29] predict strong platinum segregation in the CoPt alloy.

In this work, three indications of the AR-XPS analysis of the CoPt(30at\%) alloy equilibriated at $1000 \mathrm{~K}$ in UHV showed the platinum enrichment of the surface content. The importance of the prediction can have consequences in catalysts preparation work. 


\section{Acknowledgments}

This study was performed through Active Nano-Characterization and Technology Project, Special Coordination Funds of the Ministry of Education, Culture, Sports, Science, and Technology of the Japanese Government.

\section{References}

[1] U. Bardi, Rep. Prog. Phys. 57, 939 (1994).

[2] J.A. Rodriguez, Surf. Sci. Rep. 24, 223 (1996).

[3] M.A. Vasiliev, J. Phys. D, Appl. Phys. 30, 3037 (1997).

[4] J. Valentin, Th. Kleinefeld, D. Weller, Appl. Phys. 75, 7102 (1994).

[5] M. Jost, J. Heimel, T. Kleinefeld, Phys. Rev. B 57, 5316 (1998).

[6] M. Hansen, Constitution of Binary Alloys, McGraw-Hill, New York 1965.

[7] U. Bardi, B.C. Beard, P.N. Ross, J. Vac. Sci. Technol. A 6, 665 (1988).

[8] M. Bugnard, Y. Gauthier, R. Baudoing-Savois, Surf. Sci. 344, 42 (1995).

[9] S. Lee, Y. Okawa, Surf. Sci. 338, L833 (1995).

[10] K.S. An, A. Kimura, K. Ono, N. Kamakura, A. Kakizaki, C.Y. Park, K. Tanaka, Surf. Sci. 401, 336 (1998).

[11] Y. Gauthier, R. Baudoing-Savois, J.M. Bugnard, W. Hebenstreit, M. Schmid, P. Varga, Surf. Sci. 466, 155 (2000).

[12] T.X. Carroll, M.R.F. Siggel, T.D. Thomas, J. Electr. Spectr. Rel. Phenom. 46, 249 (1988).

[13] D. Fujita, K. Yoshihara, Surf. Interface Anal. 21, 226 (1994).

[14] J.M. Conny, C.J. Powell, L.A. Currie, Surf. Interface Anal. 26, 939 (1998).

[15] D.L. Adams, unpublished, University of Aarhus, Aarhus 2002.

[16] Practical Surface Analysis, Eds. D. Briggs, M.P. Seah, Vol. 1, Wiley, New York 1990 .

[17] C.D. Wagner, A.V. Naumkin, A. Kraut-Vaas, J.W. Allison, C.J. Powell, J.R. Rumble Jr, NIST Standard Reference Database 20 (NIST X-ray Photoelectron Spectroscopy Database), Version 3.2 (Web Version), http://srdata.nist.gov/xps/index.htm.

[18] C.J. Powell, M.P. Seah, J. Vac. Sci. Technol. A 8, 735 (1990).

[19] B. Johansson, N. Mårtensson, Phys. Rev. B 21, 4427 (1980).

[20] N. Mårtensson, R. Nyholm, H. Calén, J. Hedman, B. Johansson, Phys. Rev. B 24, 1725 (1981).

[21] P. Steiner, S. Hüfner, N. Mårtensson, B. Johansson, Solid State Commun. 37, 73 (1981).

[22] S. Doniach, M. Sunjic, J. Phys. C 3, 285 (1970).

[23] S. Hufner, G.K. Wertheim, Phys. Rev. B 11, 678 (1975).

[24] C.J. Jenks, S.L. Chang, J.W. Anderegg, P.A. Thiel, D.W. Lynch, Phys. Rev. B 54, $6301(1996)$. 
[25] F.F. Abraham, C.R. Brundle, J. Vac. Sci. Technol. 18, 506 (1981).

[26] S. Mukherjee, J.L. Moran-Lopez, Surf. Sci. 189/190, 1135 (1987).

[27] P.M. Ossi, Surf. Sci. 201, L519 (1988).

[28] A. Christensen, A.V. Ruban, P. Stoltze, K.W. Jacobsen, H.L. Skriver, J.K. Nørskov, F. Besenbacher, Phys. Rev. B 56, 5822 (1997).

[29] A.V. Ruban, H.L. Skriver, J.K. Nørskov, Phys. Rev. B 59, 15990 (1999). 